\title{
ISCHAEMIA-REPERFUSION INJURY TO THE GUT FOLLOWING CARDIOPULMONARY RESUSCITATION
}

\author{
Shrestha B M*, Throssell D
}

\section{ABSTRACT}

Ischaemia-reperfusion injury (IRI) to the gut following cardiopulmonary resuscitation (CPR) gives rise to local effects including altered mucosal permeability and infarction of the gut, and remote consequences which may culminate in multiple organ failure with a potentially fatal outcome. We describe two cases of IRI to the gut following CPR in patients where diarrhoea and abdominal pain in the first, and haematemesis, rectal bleeding and peritonitis in the second patient were the presenting features. The relevant aspects of pathophysiology and management are summarised.

IRI to the gut, defined as tissue damage occurring as a direct consequence of revascularisation, results from intestinal hypoperfusion due to cardiogenic, septic, or haemorrhagic shock, the use of vasoactive drugs, supra-coeliac aortic cross-clamping, construction of free intestinal grafts, or a mesenteric embolic event. ${ }^{1}$ We describe two cases of IRI to the gut following CPR and summarise the relevant aspects of its pathophysiology and management.

\section{Key Words: Ischaemia-reperfusion injury, gut, cardiopulmonary resuscitation.}

\section{CASE 1}

A 72-year-old male, who was on haemodialysis for an endstage renal failure of uncertain aetiology, was admitted following CPR for respiratory arrest and bradycardia. Three hours later, he complained of abdominal pain associated with profuse watery diarrhoea. Abdominal examination revealed mild generalised tenderness. There was leucocytosis (23X10\%/ L), raised serum aspartate amino transferase (1223 IU/L) and severe metabolic acidosis (blood pH: 7.2, and base deficit: 16.4). Culture of the faeces excluded infective aetiology of the diarrhoea. The patient made a full recovery with supportive therapy.

\section{CASE 2}

A 65-year-old female, who was known to suffer from hypertension and ischaemic heart disease, was admitted to the intensive care unit following CPR for ventricular fibrillation and arrest. Endotracheal intubation, ventilation and ionotropic support was required initially. Arterial blood gases demonstrated severe metabolic acidosis (blood $\mathrm{pH}$ : 6.9, and base deficit: -27). Six hours later, she vomited $300 \mathrm{mls}$ of fresh blood and passed fresh blood per rectum without alteration in haemodynamic status. Abdominal examination revealed mild generalised tenderness. With supportive treatment and enteral feeds, she made a rapid recovery with

* Sheffield Kidney Institute, Northern General Hospital, Herries Road, Sheffield S5 7AU, UK

Address for correspondence : Dr. Badri Man Shrestha

Sheffield Kidney Institute, Northern General Hospital, Herries Road, Sheffield S5 7AU, UK

Email: shresthabm@doctors.net.uk 
no further episodes of gastrointestinal (GI) bleeding. Two weeks later, she developed features of peritonitis due to perforation of the caecum, which was treated by right hemicolectomy and formation of ileostomy. This was followed by a progressive improvement in her condition, and a reversal of ileostomy is planned.

\section{DISCUSSION}

Both cases illustrate examples of IRI to the gut following CPR, where diarrhoea, severe metabolic acidosis and elevation of AST in the first, and bleeding from the GI tract and caecal perforation in the second patient, were predominant manifestations. The intestinal mucosa is particularly susceptible to IRI because of the specific microvascular anatomy, where microvilli, which constitute a large absorptive surface area, suffer most from the effects of shunting of blood from their bases during cardiac arrest. Structural changes include swelling of the organelles and the entire cell due to the entry of water and electrolytes. Lysosomal rupture precedes cell death. ${ }^{1}$

Initially, IRI is mediated by reactive oxygen free radicals and, at a later stage, by the priming and activation of polymorphonuclear neutrophils (PMN). Ischaemia and hypoxia result in depletion of cellular adenosine triphosphate (ATP), which leads to impairment of the function of sodiumpotassium-ATPase and calcium-magnesium ATPase system, thereby allowing sodium, chloride, calcium and water to diffuse into the cell and potassium and magnesium to diffuse out. The cells become oedematous and swollen and activation of cytosolic phospholipase A by calcium leads to lysis of cellular and subcellular membranes, particularly those of lysosomes. ${ }^{2}$

Hypoxia leads to degradation of ATP to adenosine monophosphate (AMP), which is catabolised further to hypoxanthine and xanthine in all ischaemic cells including those of microvilli, blood cells trapped in the vasculature and endothelial cells. Reperfusion with oxygenated blood leads to generation of superoxide free radical, hydrogen peroxide and hydroxyl free radicals and these are responsible for IRI through lipid peroxidation of cellular and subcellular membranes. Injury to the vascular endothelial cell lining favours adhesion of leucocytes, platelets and erythrocytes, thereby promoting thrombosis and secondary hypoxia. ${ }^{3}$

IRI increases intestinal mucosal permeability and promotes bacterial translocation from the intestine to the blood stream thereby leading to remote organ injury. Intestinal IRI is associated with acute lung injury and acute respiratory distress syndrome, which is the result of activation of elastase activity in the circulating neutrophils. This causes proteolysis of endothelial cell cadherins thereby disrupting the microvascular integrity in the lung. ${ }^{4}$

Management of patients with IRI should take place in a highdependency area with involvement of a multidisciplinary team consisting of intensivist, physician, surgeon, dietician, pharmacist and physiotherapist, which leads to an improved outcome. Enteral nutrition has been shown to have significant beneficial effect on mucosal immunity, gut-associated lymphoid tissue (GALT), intestinal cytokine levels and mucosal $\operatorname{IgA}$ release, thereby improving the survival rate. Conversely, TPN has been shown to exert immunosuppressive effects, such as the increased expression of interstitial cell adhesion molecule type 1, E-selectin, and P-selectin contributing to the sequestration of neutrophils in the intestine and should therefore be avoided if possible. ${ }^{5}$ Several questions in the management of IRI remain unanswered and research in this area is exploring the possible therapeutic role of induction of neutropenia, inhibition of inducible nitric oxide synthase (iNOS), administration of free radical scavengers, antileukocyte adhesion monoclonal antibodies and anti-cytokine antibodies.

In conclusion, IRI to the gut should be considered if abdominal pain, diarrhoea, bleeding per rectum and alteration of hepatic enzymes develop following CPR, which usually resolves with improvement in tissue oxygenation. Constant vigilance for the development of intra-abdominal sepsis from silent perforation of ischaemic bowel and a low threshold for laparotomy should be maintained throughout.

\section{REFERENCES}

1. Song SE, Blennerhassett IR, Heel KA, McCauley RD, Hall JC. Ischaemia-reperfusion injury to the intestine. Aust NZ Surg $1998 ; 68(8): 554-61$.

2 Homer-Vanniasinkam S, Crinnion JN, Gough MJ. Postischaemia organ dysfunction: a review. Eur J Vasc Endovasc Surg 1997; 14 (3) : 195-203.

3. Schmidt $H$, Martindale $R$. The gastrointestinal tract in critical illness. Curr Op Clin Nutr Met Care2001; 4 (6) : 547-51.

4. Carden D, Xiao F, Moak C, Willis BH, Robinson-Jackson S, Alexander S. Neutrophil elastase promotes lung microvascular injury and proteolysis of endothelial cadherin. Am J Physiol 1998; 275: 385-92.

5. Ikeda S, Zarzaur BL, Johnson CD, Fakatsu K, Kudsk KA. Total parenteral nutrition supplementation with glutamine improves survival after gut ischaemia/reperfusion. JParenteral Nutr 2002; $26(3): 169-73$. 